\title{
The validation analysis of the INSHORE system-a precise and efficient coastal survey system
}

\author{
Paulo Renato Baganha Baptista • \\ Cristina Bernardes • Telmo R. Cunha
}

Received: 8 March 2010 / Accepted: 21 October 2010

(C) Springer Science+Business Media B.V. 2010

\begin{abstract}
Government and environmental entities are becoming increasingly concerned with qualifying and quantifying the erosion effects that are observed in sandy shores. Correspondingly, survey methodologies that gather data for such erosion studies are increasingly being demanded. The responsible entities are continually broadening their areas of interest, are concerned in the establishment of regular monitoring programmes and are demanding high accuracy from the geospatial data that is collected. The budget available for such monitoring activities, however, does not parallel the trend in the increasing demand for quality specifications. Survey methodologies need improvement to meet these requirements. We have developed a new land-based survey systemthe INSHORE system-that is ideal for low cost,
\end{abstract}

P. R. Baganha Baptista $(\varangle) \cdot$ C. Bernardes

Department of Geosciences, Centre

for Environmental and Marine Studies (CESAM),

University of Aveiro, Campus Universitário

de Santiago, 3810-193, Aveiro, Portugal

e-mail: renato.baganha@ua.pt

T. R. Cunha

Department of Electronics, Telecommunications and Informatics, Institute of Telecommunications, University of Aveiro, Campus Universitário de Santiago, 3810-193, Aveiro, Portugal highly efficient and highly precise coastal surveys. The INSHORE system uses hi-tech hardware that is based on high-grade global positioning system (GPS) receivers and a laser distance sensor combined with advanced software algorithms. This system enables the determination of the ground coordinates of the surveyed areas with a precision of 1 to $2 \mathrm{~cm}$, without having a sensor in contact with the ground surface. The absence of physical contact with the ground makes this system suitable for high-efficiency surveys. The accuracy of the positioning, which is based on advanced differential GPS processing, is enhanced by considering the estimated attitude of the GPS receiver holding structure and eliminates undesirable offsets. This paper describes the INSHORE survey system and presents the results of validation tests that were performed in a sandy shore environment.

Keywords Topography • DGPS • Survey system • Attitude - Accuracy

\section{Introduction}

An awareness of climatic changes and their consequences has led the responsible authorities of several governments with sandy shore borders to create and implement coastal management plans that will avoid drastic and sudden erosion effects. 
In countries such as Portugal, where about half of the political border is constituted by sandy shores, special attention must be paid to the temporal changes of these littoral features. To perform an accurate assessment of sandy shores, the subaerial monitoring methodologies employed at the beach must meet strict specifications, including the following: (1) low operation costs; (2) highly efficient surveying; (3) high accuracy in the determination of the geo-spatial morphological elements of the terrain and the associated physical parameters, such as beach volumes and (4) the ability to perform frequent monitoring campaigns (at time scales ranging from days to weeks, or months) over stretches of beach tens of kilometres long. These specifications are demanding on the survey methodology.

The main geo-spatial information that is required for sandy shore analysis is the volumetric quantity of sediments within a specified study area. The seasonal variation in this data provides information on the beach dynamics. Shoreline evolution, measured in the form of the frontal dune baseline or other shoreline indicators, is also important as it provides information on the retreat or advancement of the shore. It therefore seems reasonable that dedicated survey procedures would be able to determine the threedimensional coordinates of ground points with an altimetric accuracy below $3 \mathrm{~cm}$ for standard monitoring scales. The requirement of high altimetric accuracy is due to the rising offsets in the calculation of sediment volume (because this calculation is performed with the mathematical operation of integration). The local vertical component must be accurate and must not contain a systematic offset (i.e. its error must be randomly distributed around zero). After integration, such an offset would lead to a significant offset in the calculation of the volume. The offset could also be misleading in the analysis of volumetric variation between consecutive monitoring events.

Since the 1960s, many different survey methodologies and techniques have been developed and published. The following is a brief description of some of the techniques that are specific to volumetric quantification and shoreline delineation. Traditionally, sandy shore survey were made through cross-shore profiles carried out with theodolites and total stations. These applications require at least two operators; one for the total station and the other holding the target steadily at every point that is to be coordinated (Emery 1961; Komar 1976). This method, although accurate (in terms of coordinate determination), is not suited for the frequent monitoring of long coastal stretches due to its inefficiency (Dornbusch et al. 2008).

Another technology that has been used successfully in several applications is image processing (digital or analog). It is possible to generate digital elevation models (DEM) that map the coastal surface based on airborne photogrammetry with sequential and overlapping high-resolution photos (Fletcher et al. 2002; Anders and Byrnes 1991). However, to achieve accuracy of a few centimetres it is necessary to have the coordinates of about six ground points per photo (the socalled ground control points, or the GCPs). These are the points that are determined through static mode differential global positioning system (GPS) surveying. Though this method has good accuracy, it is expensive due to the equipment required for each survey, and it is not efficient as the survey of the GCPs is time consuming. Airborne photogrammetry requires meteorological conditions that are not always compatible with post-storm beach surveys. Moreover, on sandy surfaces is it not easy to identify good candidates for GCPs because these sites must be easily identified on the photo and must be well dispersed in each photo; the homogeny of the sandy surface makes this a difficult task. An alternative to airborne photogrammetry is the airborne laser scanning technique, which is efficient and provides reasonable accuracy (Huising and Gomes Pereira 1998; Shrestha et al. 2005). However, the requirement of an aircraft and the expensive laser scanner makes this solution unattractive from the perspective of the operation costs. A different approach to image-based coastal monitoring is the use of fixed high-resolution digital cameras installed along the coastal stretch (Lippmann and Holman 1989; Holman et al. 1993). With cameras mounted on the top of high poles, information regarding shore dynamics can be determined through the analysis of images captured at different points in time. However, the precision obtained with this method 
is not at the centimetre level, and it drastically deteriorates with the distance to the camera. Additionally, the cost and effort required to use this technique on the long shore stretch could be considerable due to the required infrastructures and due to vandalism preventive actions.

More recently, new terrestrial-based techniques have been used, with a special emphasis on those based on GPS positioning. With an overall accuracy around $2 \mathrm{~cm}$ in the kinematic mode (James and Farrell 1976), high-grade GPS receivers can deliver precise positions at rates of one position per second or higher (HofmannWellenhof et al. 2001). Thus, fixing a GPS antenna to a vehicle suited for sandy surfaces, such as a motor-quad, leads to an efficient method for shoreline delineation. By using a land-based platform with a GPS survey system, data can also be acquired through beach profiles performed in along-shore and cross-shore transects, making a grid whose mesh is adopted according with the morphological characteristics of the sandy shore. From that data DEMs are generated by using interpolation procedures, allowing afterwards computing the beach volume. It is essential that the sources of the data-the GPS field positions-are as accurate as possible, especially in the vertical component. In this context, different approaches can be found in literature. For instance, single GPS antenna solutions were published in (Morton et al. 1993) and (Haxel and Holman 2004) where a fixed estimate of the antenna height to the ground was subtracted from the GPS antenna coordinates along the local vertical direction. Some recent sandy shore research activities are based in this kind of survey system (Barnard and Warrick 2010; Hansen and Barnard 2010). Other techniques report the use of multi-antenna GPS systems that try to compensate the vertical deviations of the GPS antennas that are due to the changes in the shore surface slope (Groat 2000; List et al. 2006; Baptista et al. 2008). As stated in List et al. (2006) and in Baptista et al. (2008), this is an improvement on the single-antenna solutions because the vehicle supporting the antenna does change is attitude during the survey. If the antenna height is directly subtracted from the antenna coordinates in the vertical direction then the systematic offsets will be produced in the final solutions. The multi-antenna system tries to compensate such offsets. However, two variables are not properly accounted for in these proposed strategies. First, the attitude of the vehicle supporting the antennas (with respect to a local reference frame) does not always coincide with the slope of the surface due to the vehicle suspension mechanism. Second, the slope height of the GPS antenna phase centre (the one used for coordinate reference) varies along the survey. Several causes contribute to this variation (1) the suspension mechanism of a vehicle that is suited for sandy surfaces is far from being rigid, thus the distance of the chassis to the ground is always changing as the vehicle moves; (2) according to the vehicle dynamics, and also to the composition of the surface materials, the interaction between the tires of the vehicle and the sandy surface is constantly changing, causing slope height changes and (3) other phenomena such as tire pressure variations with temperature and usage may also lead to systematic errors. The magnitude of these systemic errors in terms of the slope height can easily reach $10 \mathrm{~cm}$ or more in the vertical component (Lancker et al. 2004), thus inducing significant offsets in the volumetric analysis.

With the above issues in mind, a multi-antenna system was proposed in Baptista et al. (2008, 2010) that accounted for the attitude of the structure holding the reference GPS antenna, and that kept the slope height of the antenna constant with respect to the ground. This was accomplished by adding a degree of freedom into the movement of the system structure, allowing its roll angle to change with respect to the vehicle attitude, and adding a contact point between the structure and the ground surface, by means of an extra wheel. Baptista et al. $(2008,2010)$ demonstrated that the high accuracy that is obtainable with such a system. However, this system requires that the extra wheel that is attached to the structure is always in contact with the ground surface, which imposes an upper limit on the velocity of the survey vehicle (and depends on the roughness or smoothness of the surface).

To avoid this limitation on the efficiency of the survey method proposed in Baptista et al. (2008, 2010), a new survey system was designed and implemented and is now presented in this 
paper. Contrary to the system presented in Baptista et al. (2008, 2010), this new system, named as INSHORE (INtegrated System for High Operational REsolution in shore monitoring), is based on a structure that is rigidly fixed to the survey vehicle (a motor-quad), and, instead of maintaining the distance of the reference GPS antenna to the ground as a constant, it allows it to change. A laser-based high-accuracy distance sensor is used to monitor such variations. In this manner, the survey velocity becomes limited only by the restrictions imposed by the vehicle itself or by terrain characteristics, and not by the survey system equipment.

The INSHORE survey system is described in "Material and methods". The evaluation of the INSHORE system accuracy and its performance is assessed in "Results", with concluding remarks presented in "Discussion".

\section{Material and methods}

The INSHORE survey system consists of a metallic structure that holds a set of sensors. The objective of the system is to determine, with a high level of accuracy, the three-dimensional coordinates of points in the ground surface. The structure has a triangular shape with two vertices fixed along the side of a motor-quad; the third vertex points horizontally out of the vehicle. Over each of the two inner vertices, a single frequency (L1) GPS antenna is connected to two low-grade GPS receivers (that store the raw data received from the GPS satellites). On the outer vertex, there is a dual-frequency GPS antenna connected to a highgrade GPS receiver; this antenna is the one that is used as the coordinate reference. A fourth GPS receiver is installed over a fixed point near the survey site so that differential GPS processing can be performed. The positions of the phase centre of the outer antenna are then determined through dedicated GPS processing software, fixing the L1 ambiguities, which lead to instantaneous positions with an accuracy on the 2-cm level (James and Farrell 1976). The two low-grade GPS receivers and the high-grade receiver are used to estimate the inclination angles (i.e. the attitude) of the triangular structure for each sample period. The three-dimensional vectors from the inner antenna on the back to the other two antennas are determined using the software that was specially developed for this purpose. The roll, pitch and heading (or yaw) angles of the structure are then calculated with respect to a local reference frame. This specific software also used L1 ambiguity fixing to determine highly accurate vectors, providing a good estimate of the attitude of the structure at each sampling.

Below the outer dual-frequency GPS antenna is a laser distance sensor that measures the vertical distance to the ground if the structure is levelled, otherwise it measures the slope distance to the ground. A high-grade laser sensor was chosen, with a sampling time that had less than 1 millisecond and better than $1 \mathrm{~cm}$ accuracies when reflecting from a sandy surface.

Thus, with the high accuracy of coordinates of the outer GPS antenna, the direction of the laser beam (which is given by the structure attitude estimate after geometric calculations), the distance from the laser to the ground and the coordinates of the ground point where the laser is reflecting can be easily determined through geometry analysis (i.e. the application of rotation matrices). Figure 1 shows a picture of the INSHORE system mounted on the motor-quad vehicle, with every major component identified.

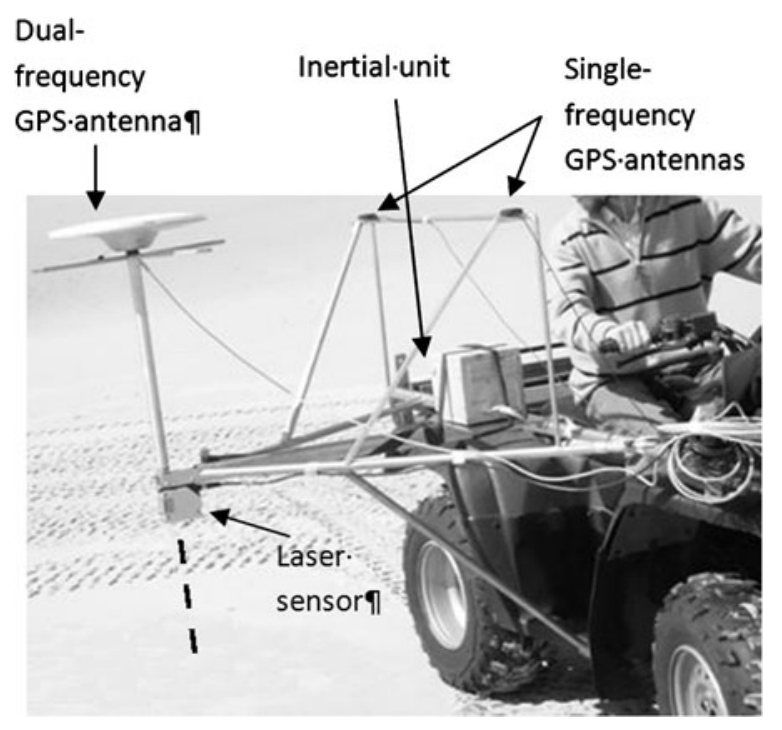

Fig. 1 Image of the INSHORE survey system, mounted on a motor-quad vehicle 
Fig. 2 Experimental beach area (Aveiro, Portugal)

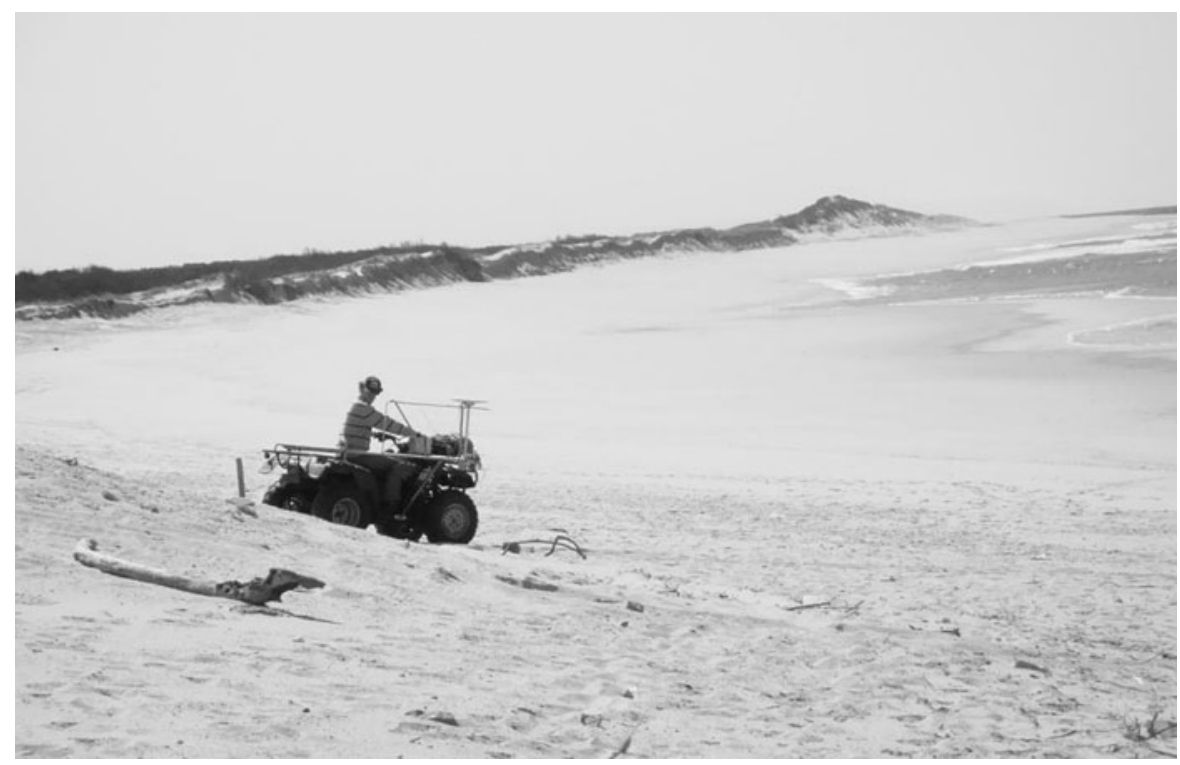

As can be seen in Fig. 1, an inertial sensor unit is also attached to the metallic structure. This unit contains a set of three gyroscopes displaced along three orthogonal directions, with one accelerometer in each of those directions. The inertial unit measures the linear and the rotational accelerations of the structure, from which an estimation of the structure attitude angles can be inferred (James and Farrell 1976). In the case of the INSHORE system, the inertial unit was included for redundancy in the determination of the structure attitude, contributing to a robustness increase (it is very useful in detecting and compensating possible outliers of the GPS solutions due to spurious multipath reflections, noise or interference from other external devices).

Because the INSHORE system does not have a point of physical contact with the ground surface (except for the vehicle wheels) and with the high sampling rate that the installed sensors can deliver (on average four measurements per second, but it can reach ten measurements per second), it can perform surveys at high velocity. In fact, the limit on the survey velocity is now imposed by the safe operation of the vehicle, according to the morphologic characteristics of the sandy surface being surveyed. This leads to a significant improvement in efficiency in comparison to the solution presented in Baptista et al. (2008) while maintaining the same level of accuracy.

\section{Results}

After assembling the INSHORE system, four validation tests were performed to assess the accuracy and performance. This section describes the tests and presents the obtained results.

A reflective/intermediate sandy shore, with the morphodynamic classification of Wright and Short (1984), was used to test the system. The shore was located in the coastal region of Vagueira, Aveiro, Portugal, where the sandy surface included both high and low slope characteristics (Fig. 2). Twenty points were materialised on the ground through metal plates (Fig. 3) displaced on a test grid of $4 \times 5$ control points. Figure $4 \mathrm{a}$

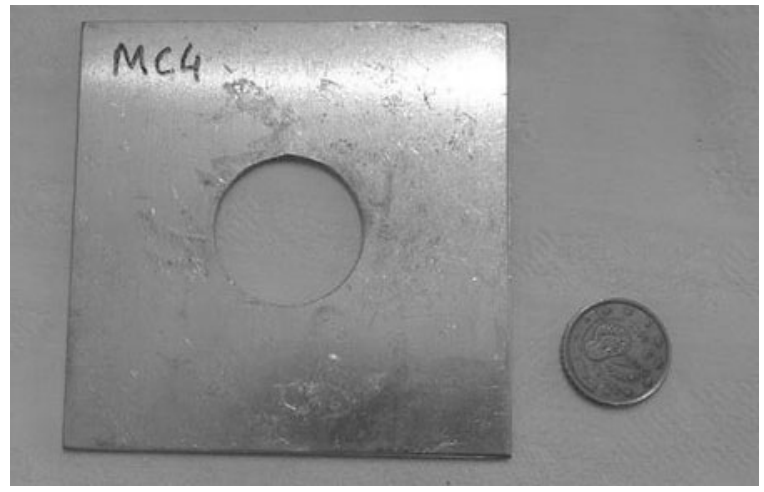

Fig. 3 Example of a metal plate used to materialise the points of the test grid 
Fig. 4 The test grid: a planimetric view; b three-dimensional view
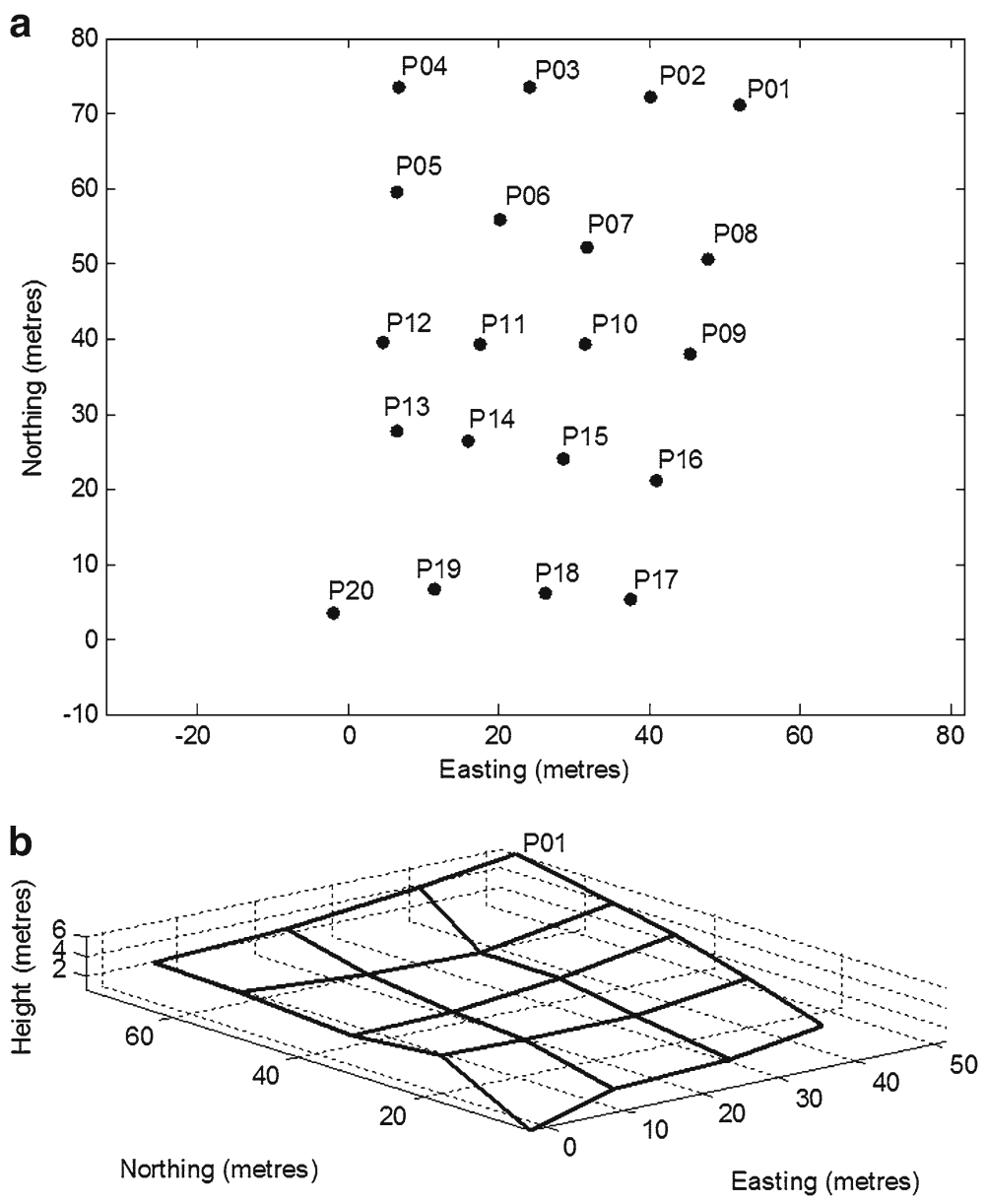

shows the planimetric view of the test grid (in a local reference plane), and in Fig. 4b, the threedimensional view of the grid is presented. The control points of the test grid were coordinated with static GPS processing using the differential mode by placing a GPS antenna in each point for a considerable time. After L1 ambiguity fixing and averaging the determined positions for each grid control point, the grid coordinates were determined to have accuracy better than $1 \mathrm{~cm}$. The test grid provided the reference points to the validation of the INSHORE system results, after it passes over the grid points.

Grid surveying in static mode with roll angle variations

The first test consisted of surveying the grid parallel to the shoreline, where the roll angle of the vehicle is the most excited. Figure 5 shows the path followed by the INSHORE system. Along the path, the system operator tried to aim the point of incidence of the laser beam close to the grid control points, stopping near each control point for a period of $10 \mathrm{~s}$. Data were recorded at a rate of two measurements per second. The recorded data were processed with dedicated software and the ground positions were determined. Information on the structure attitude was also included. The results were compared to the reference coordinates of the grid control points that were previously determined by static GPS positioning. The objective of this test was to determine the accuracy of the ground coordinates produced by the INSHORE survey system when significant variations in the roll angle were encountered. Figure 6 shows the difference in the altimetric coordinates between the reference positions and the 
Fig. 5 The path followed by the INSHORE system in the first validation test

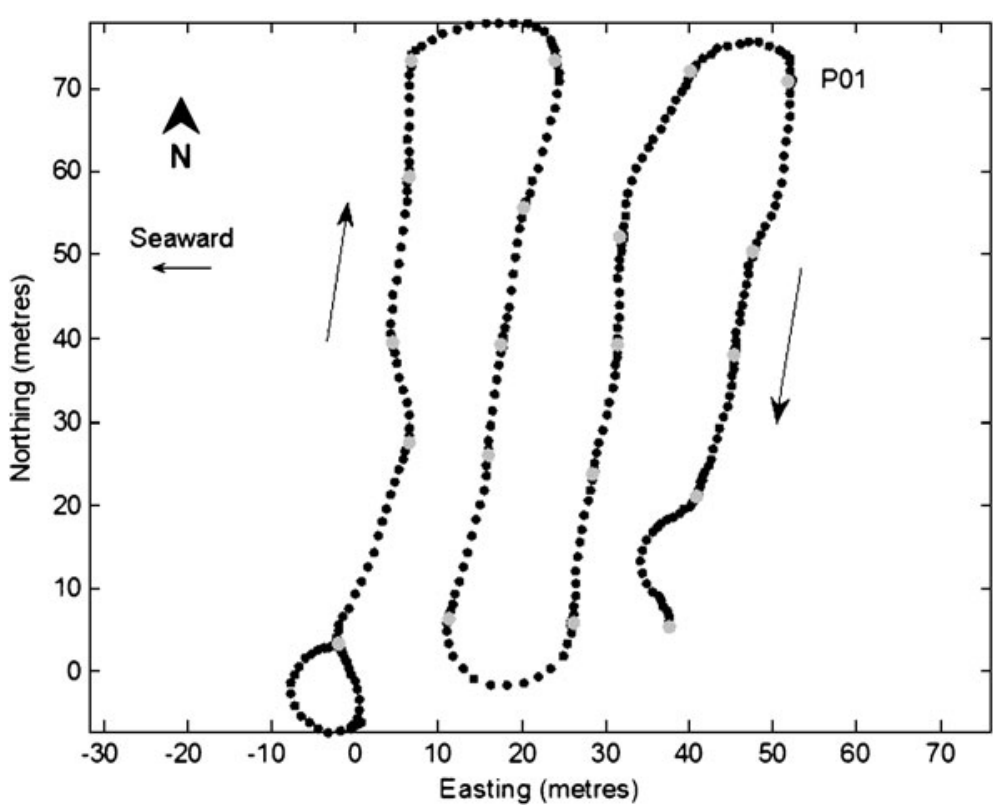

surveyed solutions. Figure 7 shows the difference in planimetry for each of the 20 control points.

The results displayed in Fig. 6 show that almost all of the points were coordinated with an error within $2 \mathrm{~cm}$ in altimetry. However, five of the points have an altimetric error slightly over $2 \mathrm{~cm}$, which is related with the higher planimetric differences between the INSHORE survey system and the grid control points, as can be observed in Fig. 7. This test was performed in conditions that were as similar as possible to the ones found during survey conducted without special vehicle driving procedures (i.e. those that make the laser bean emission point coincident to the grid control points). Because the sandy surface was not completely levelled around the metallic marks, some altimetric errors were expected (approximately 1 or $2 \mathrm{~cm}$ ). As a consequence, the INSHORE sys-
Fig. 6 The difference in altimetry between the reference positions of the grid points and the solution obtained with the INSHORE system in the first validation test

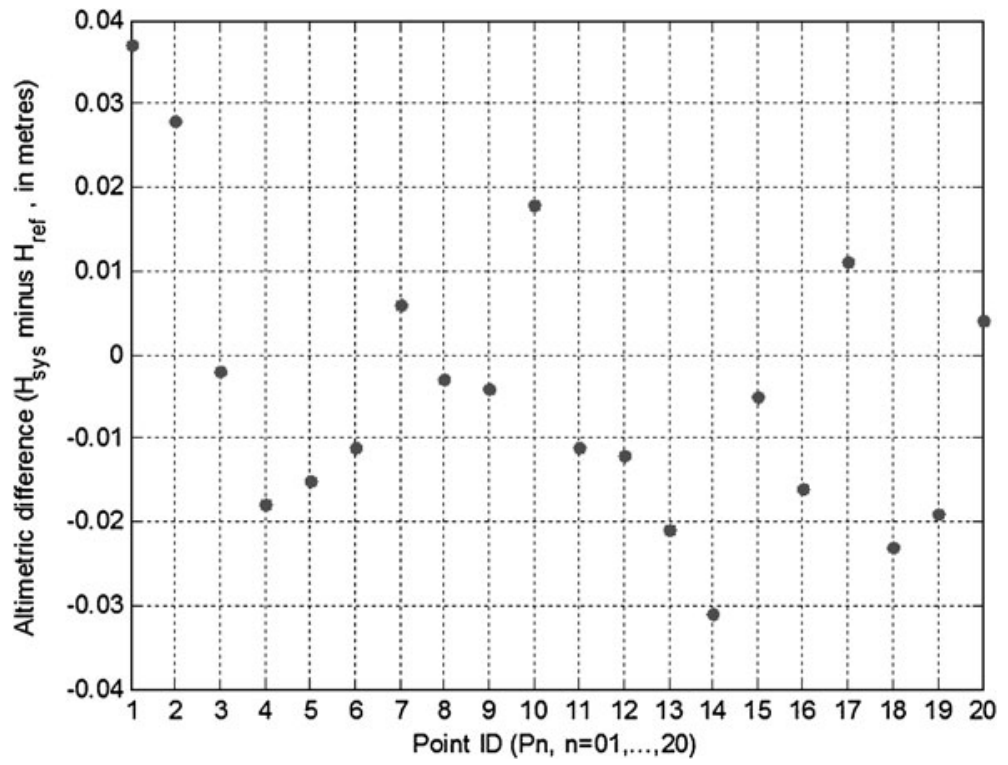


Fig. 7 The difference in planimetry between the reference positions of the grid and the solution obtained with the INSHORE system in the first validation test

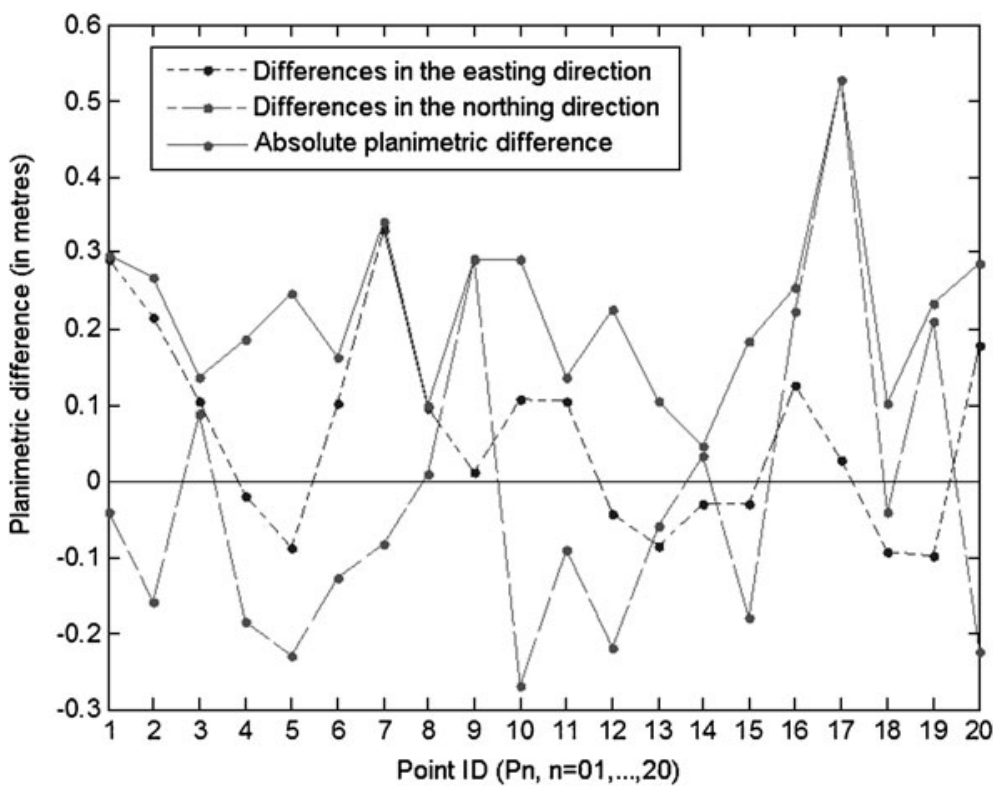

tem was never placed exactly over the metal plates materialising the grid point. Figure 7 shows that, in planimetry, the INSHORE system was around 10 to $30 \mathrm{~cm}$ away from the grid points. At the points where the surface slope was higher (i.e. those closer to the dune baseline), equal differences in planimetry caused higher errors in altimetry compared to points of lower surface slope (i.e. those near the shoreline).
To further illustrate the importance of the inclusion of the structure attitude, the distance from the dual-frequency GPS antenna (at the outer vertex) to the ground (given by the fixed and known distance from its phase centre to the laser sensor focus, plus the distance measurement to the ground surface provided by the laser) was subtracted from the coordinates of the GPS antenna phase centre. The obtained altimetric values for
Fig. 8 The effect on the altimetric component with the exclusion of the structure attitude (i.e. the difference from the altimetric height determined with the attitude estimate to that obtained with no attitude information) in the first validation test

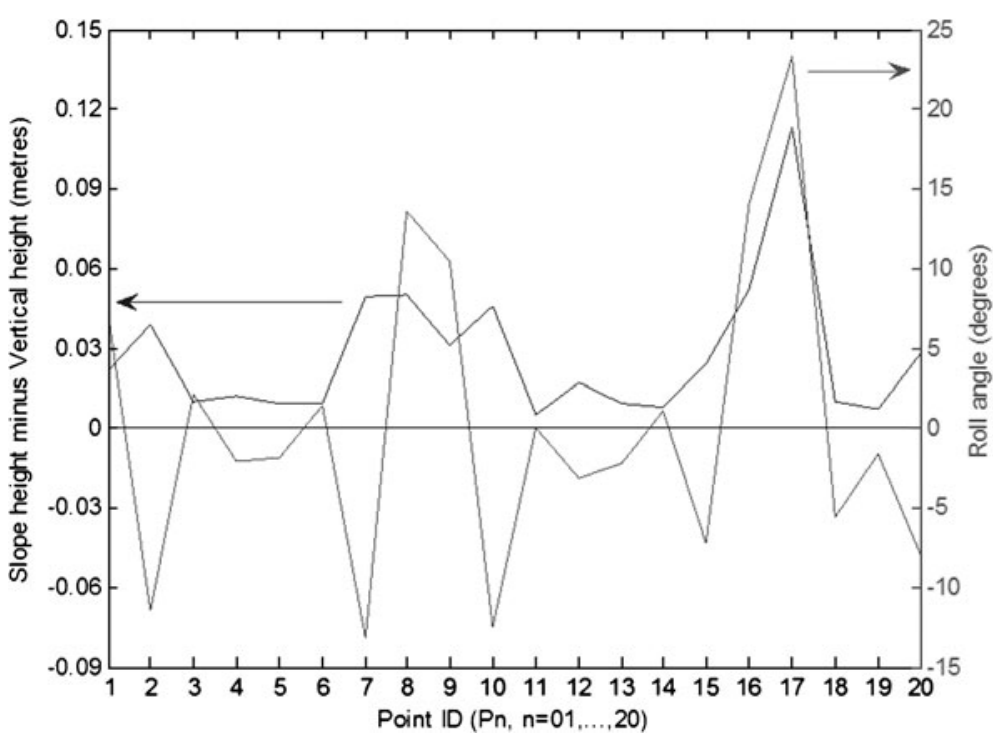


Fig. 9 The effect of the exclusion of the structure attitude (i.e. the difference from the planimetric coordinates determined with the attitude estimate to those obtained with no attitude information) on the planimetry component of in the first validation test

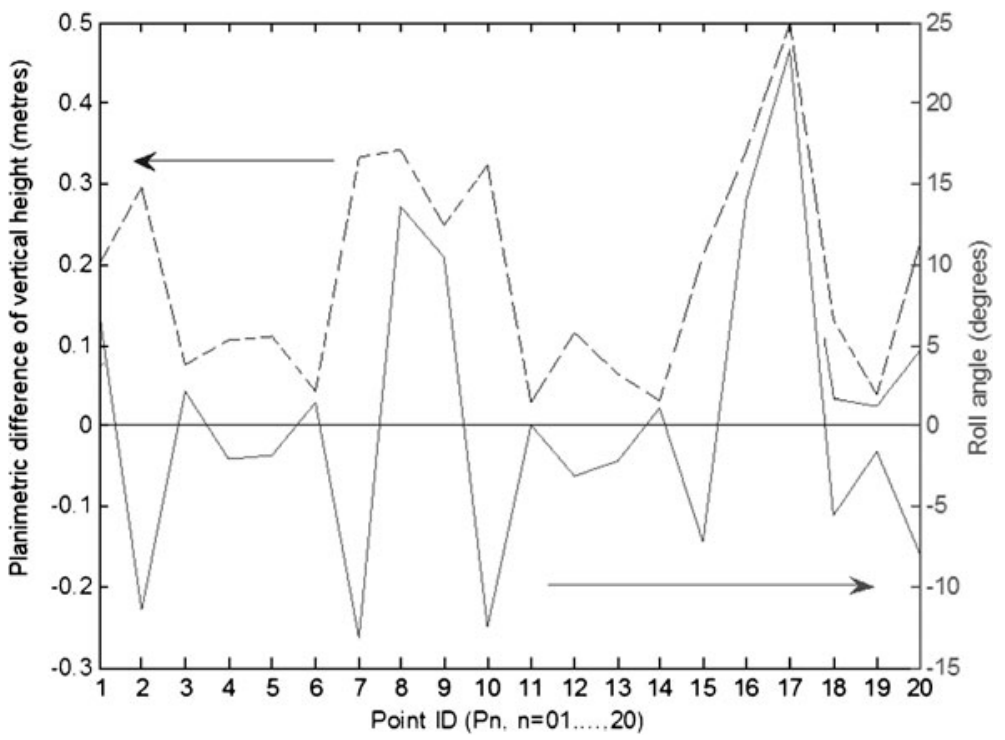

the grid control points were compared to those determined previously by the INSHORE system (which included the attitude estimate) and are depicted in Fig. 8. To better understand the origin of the differences observed in Fig. 8, the roll angle is also displayed in the graphic. In this test, this angle is more pronounced. As expected, this difference is more significant with higher roll angles; at roll values of $13^{\circ}$, the difference was about $5 \mathrm{~cm}$, and for a roll angle of $23^{\circ}$, the difference reached almost $12 \mathrm{~cm}$. These differences are considerable, and more importantly, they are systematic errors that are not equally distributed around zero, thus leading to significant offsets in the sandy shore analysis such as those seen in the estimation of the volume of the shore sediments referred to in "Introduction".

Because the system can also be used for the delineation of morphological features, the inclusion of the attitude estimation was also analysed in
Fig. 10 The path followed by the INSHORE system in the second validation test

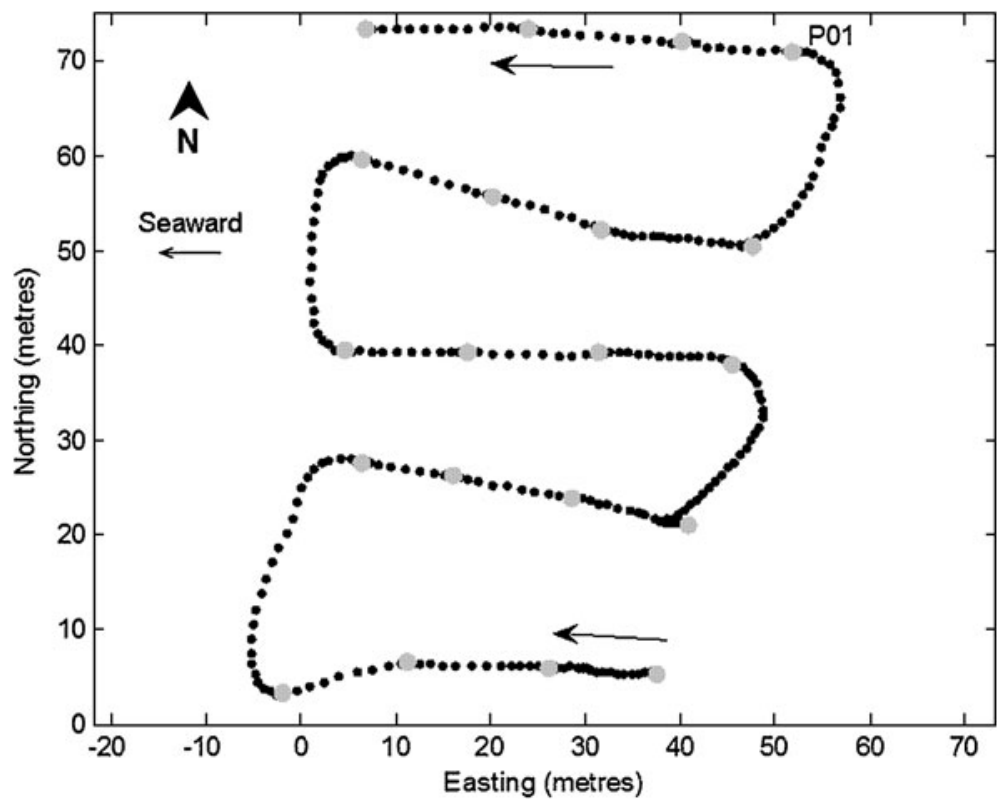


planimetry, resulting in the comparison presented in Fig. 9. Again, significant offsets are observed with respect to the results of the INSHORE system, and the strong correlation with the roll angle is again evidenced.

Grid surveying in the static mode with pitch angle variations

The second test was similar to the former test, except that the grid was now surveyed in the direction perpendicular to the shoreline. In this case, the pitch angle was the most excited angle. As before, the INSHORE system stopped for $10 \mathrm{~s}$ near each grid point. Figure 10 shows the trajectory that was followed, and in Fig. 11, the altimetric difference from the INSHORE solution is presented at each grid point relative to their reference coordinates. Once again, the accuracy of the INSHORE system in the altimetric component is shown to be better than $2 \mathrm{~cm}$, especially when considering that the laser reflection point was not exactly coincident with the grid points as can be seen in Fig. 12 where the differences in planimetry are given for each point.

The ground point solutions were computed without considering the information of the structure inclination angles by subtracting the distance of the antenna to the ground along the local vertical direction. The altimetric differences from the former solution produced by the INSHORE system (with attitude included) are shown in Fig. 13, together with the pitch angle values for the correlation analysis. In Fig. 14, the same difference is displayed, but in the planimetric coordinates. It can be observed that, even though the surveyed surface is the same as in the first test, the pitch angles in the current test did not reach values as high as the roll angle values of the first test. This result is because the width of the motor-quad vehicle is shorter than the distance between the front and rear axles, and its suspension mechanism behaves differently in the longitudinal and transversal directions, allowing higher tilt angles in the transversal or roll direction. Nevertheless, from Figs. 13 and 14 the importance of including the attitude information in the estimation of the ground points coordinates is once again demonstrated.

Grid surveying in the kinematic mode with reduced velocity

The third test consisted of the survey of the grid with the INSHORE system running at a low velocity (around $10 \mathrm{~km} / \mathrm{h}$ ) and without stopping near each point. The objective of this test was to
Fig. 11 The difference in altimetry between the reference positions of the grid points and the solution obtained with the INSHORE system in the second validation test

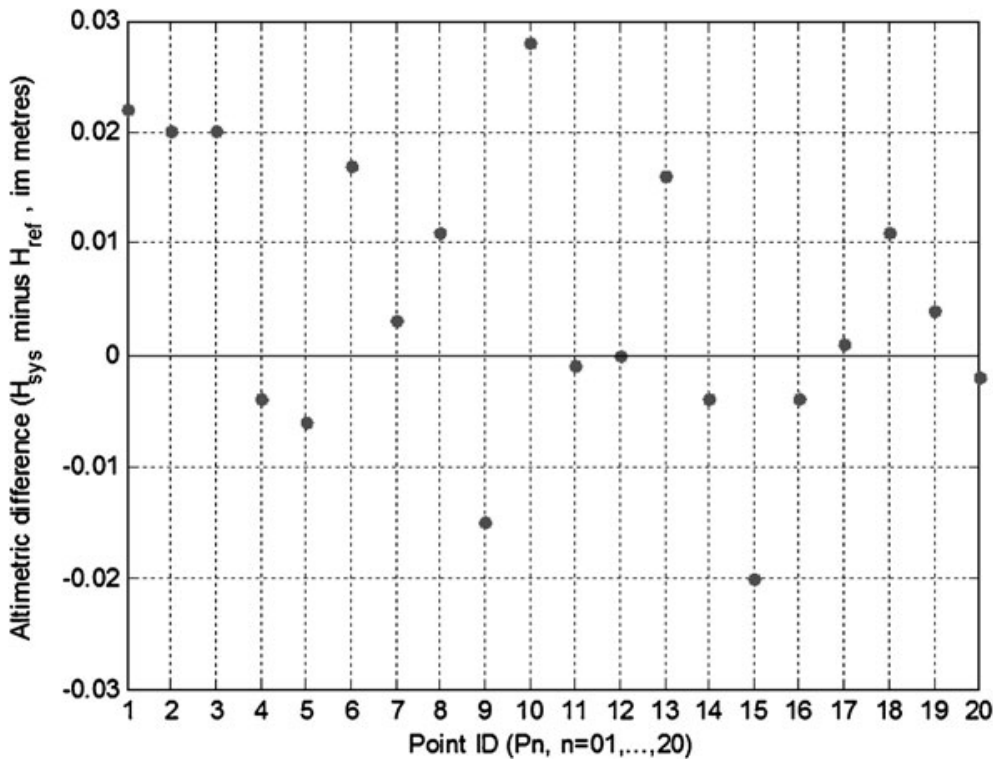


Fig. 12 The difference in planimetry between the reference positions of the grid points and the solution obtained with the INSHORE system in the second validation test

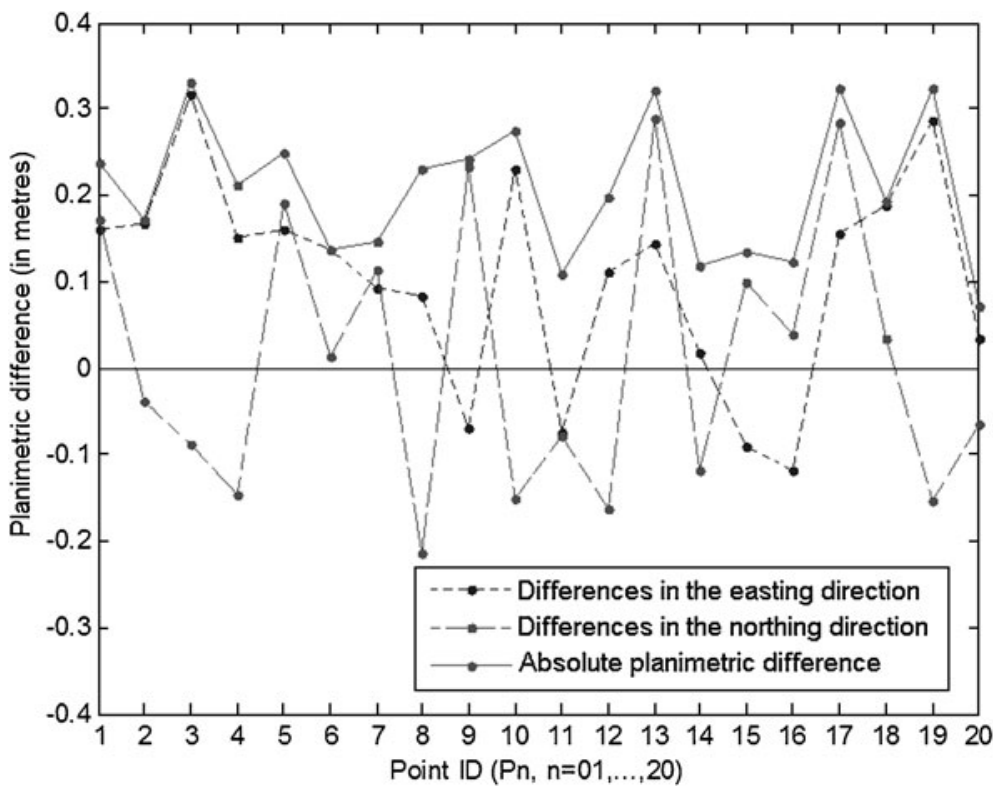

observe the INSHORE system behaviour under dynamic conditions. The system initially surveyed the grid in the direction parallel to the shoreline (as in the first test), followed by a second run in the perpendicular direction (as in the second test). Thus, the INSHORE system passed near the grid points two times with two distinct sets of attitude angles. Because it was not easy for the operator to properly identify the metallic marks on the ground nor the laser reflection point in advance and be- cause the operation constraints that naturally are more demanding with an increasing survey velocity, it was assumed that the observed differences in planimetry (and consequently, altimetry) would be higher than those observed in the previous two tests. Moreover, as the system was generating two solutions per second, it was expected that the sampling instants of the system equipment would not be coincident with the instant that it passed over the grid mark. To reduce these uncertain-
Fig. 13 The effect of the exclusion of the structure attitude (i.e. the difference from the altimetric height determined with the attitude estimate to that obtained with no attitude information) on the altimetric component in the second validation test

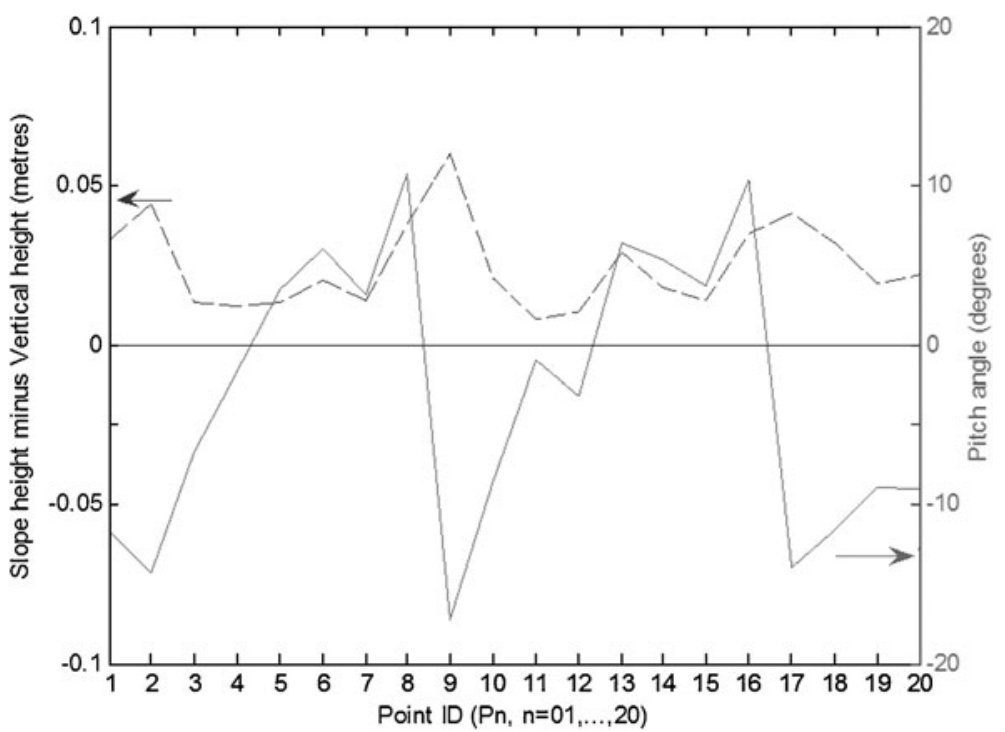


Fig. 14 The effect of the exclusion of the structure attitude (i.e. the difference from the planimetric coordinates determined with the attitude estimate to those obtained with no attitude information) on the planimetric component in the second validation test

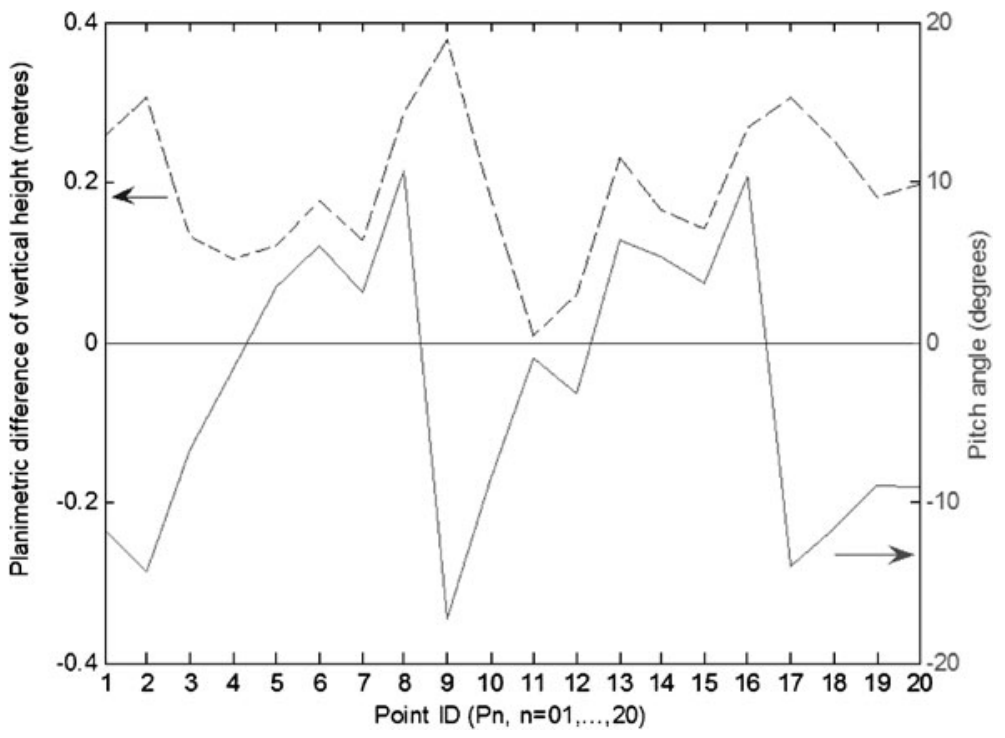

ties, the path was interpolated by means of firstorder polynomials (between consecutive points). The points of the piecewise linear track that were closest to the grid points were determined and registered. These selected points (two points per grid vertex, one for each of the runs over the grid) were then compared to the reference coordinates of the grid. Figures 15 and 16 show the results of this comparison in altimetry and planimetry, respectively. The average value of the altimetric error (considering all passages on the grid points) was $-0.6 \mathrm{~cm}$ with a standard deviation of $2.0 \mathrm{~cm}$, while for the planimetric error a mean value of $22.6 \mathrm{~cm}$ was obtained with a standard deviation of $20.0 \mathrm{~cm}$. This clearly shows the achieved high accuracy in the altimetric component (the error in the planimetric component is most entirely due to the lack of ability that the INSHORE system
Fig. 15 The difference in altimetry between the reference positions of the grid points and the solution obtained with the INSHORE system in the third validation test

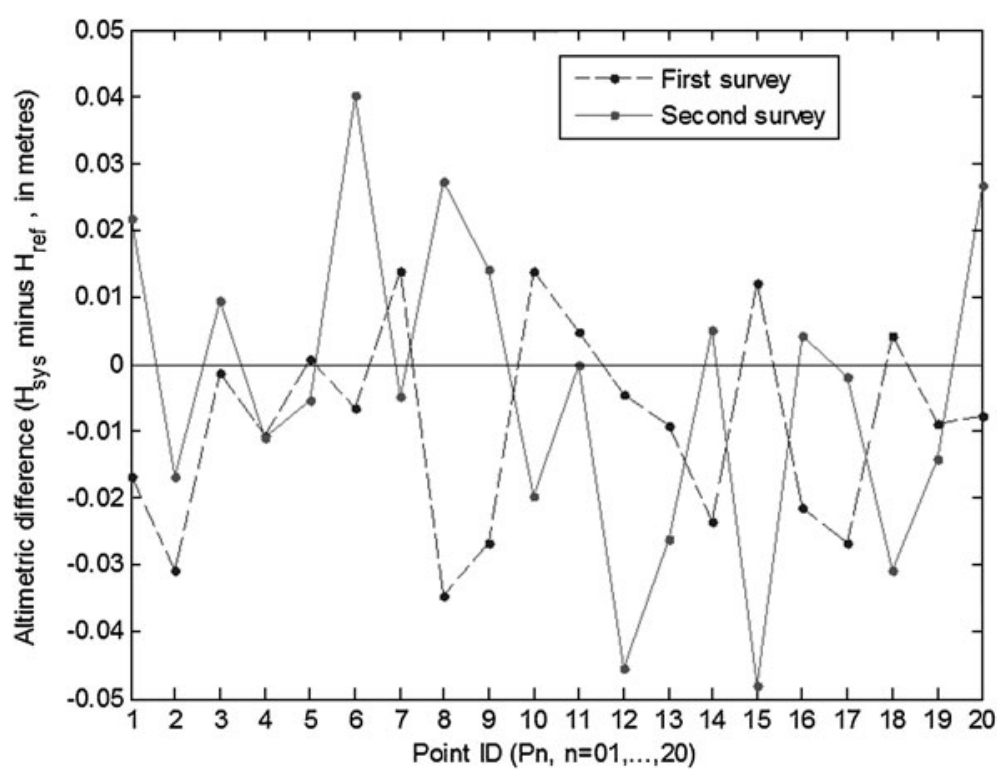


Fig. 16 The difference in planimetry between the reference positions of the grid points and the solution obtained with the INSHORE system in the third validation test

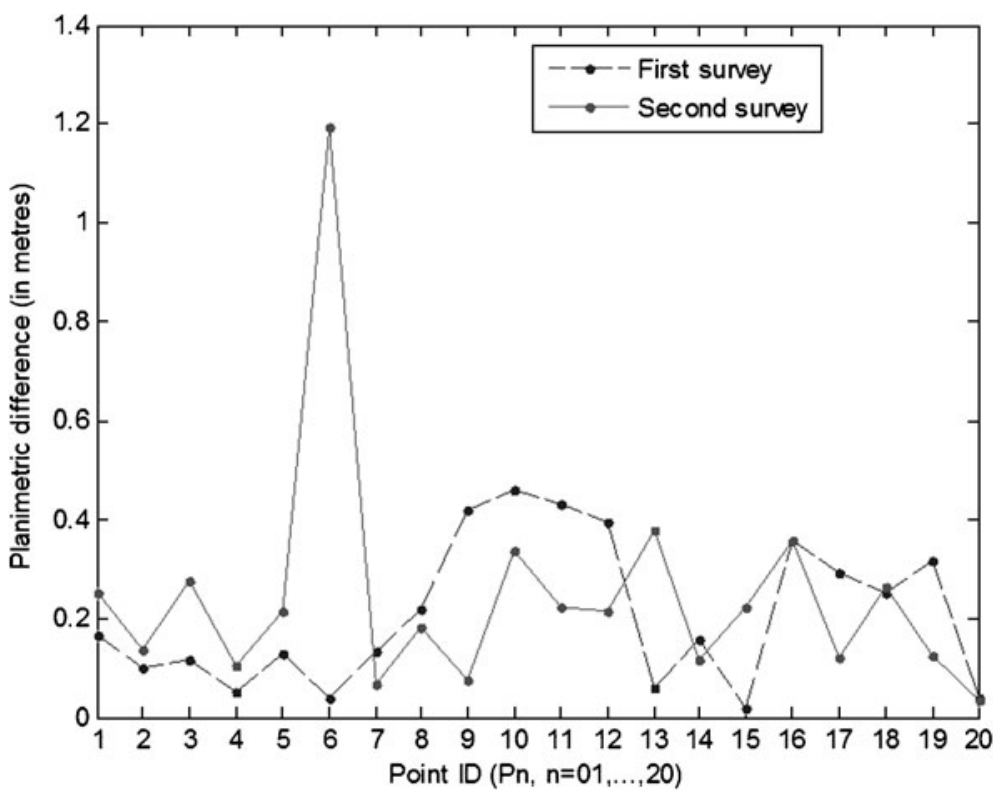

operator had in passing the laser beam exactly over the grid points).

As was expected, these differences are slightly higher than the ones found in the first two tests, however these are within the expected range, taking into account that the system did not pass directly over the grid points, as explained above. This demonstrates that the INSHORE system does not have performance degradation due to the increase of the survey velocity. The inherent difficulties result from the operation of the system and are not intrinsic to the system.
Fig. 17 The difference in altimetry between the reference positions of the grid points and the solution obtained with the INSHORE system in the fourth validation test

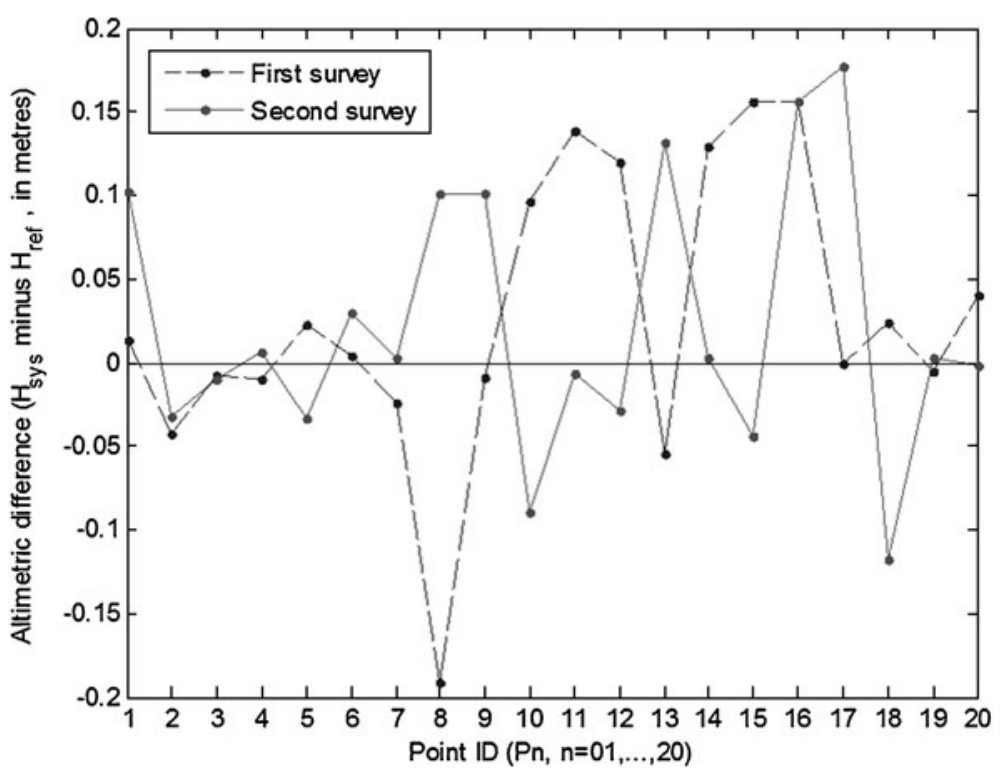


Fig. 18 The difference in planimetry between the reference positions of the grid points and the solution obtained with the INSHORE system in the fourth validation test

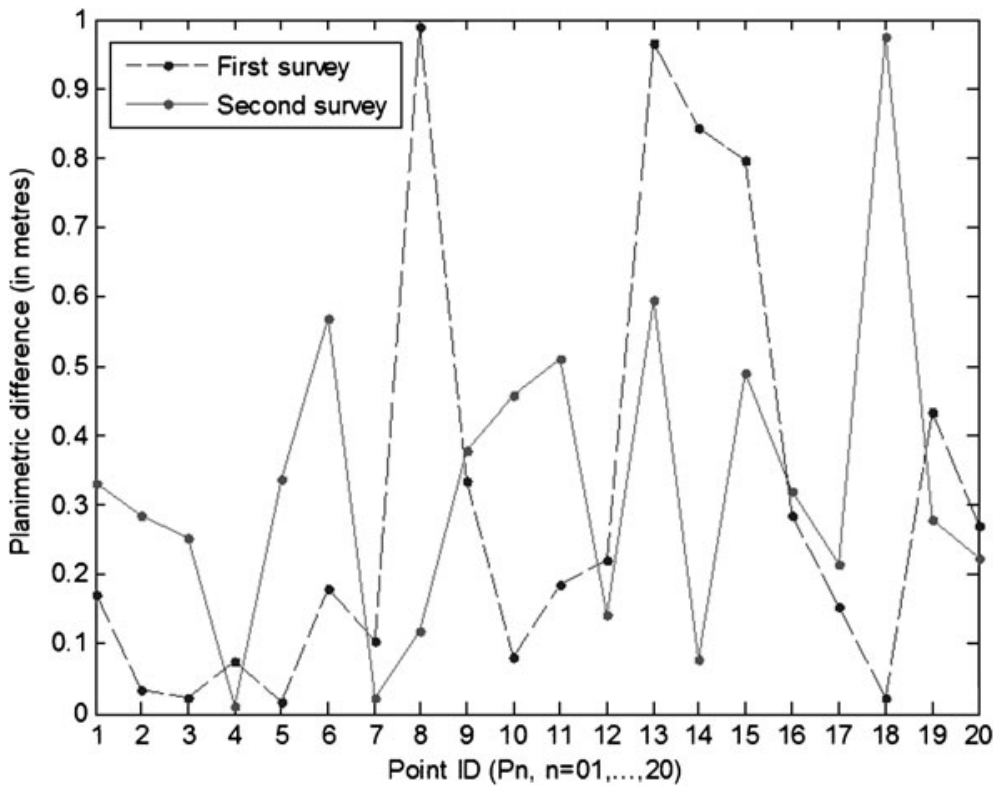

Grid surveying in kinematic mode with moderate velocity

The previous test was repeated for a survey velocity of approximately $20 \mathrm{~km} / \mathrm{h}$ (the double of that of the former test). The same analysis was performed and the results for the altimetric and planimetric differences from closest points of the piecewise linear path to the grid points are shown in Figs. 17 and 18, respectively. Because the velocity is higher, the probability of having a data sample coincident with the passage of the INSHORE system exactly over the grid points is further reduced. In this test, the average error in altimetry (considering all passages over the grid points) was $2.5 \mathrm{~cm}$ with a standard deviation of $8.2 \mathrm{~cm}$, while the planimetric error achieved a mean value of $31.9 \mathrm{~cm}$ with a standard deviation of $27.7 \mathrm{~cm}$. As expected, the altimetric errors are higher than those of the third test since the coincidence between grid points and surveyed points is much lower than in the previous tests. Operating the vehicle so that the laser beam crosses over the grid marks became even more difficult at this speed. The operator had difficulty visually identifying the metallic marks in advance on the majority of the passes. It can be seen from Figs. 17 and 18 that for points with low planimetric differences the altimetric error is still inside the $2-3 \mathrm{~cm}$ range, demonstrating that the system is also highly accurate in the kinematic mode-the normal operating mode.

\section{Discussion}

The validation tests presented in "Results" were designed with the purpose of testing the INSHORE system in the same environment found under normal operation-a sandy shore. We believe that the obtained results are closer to those that will result from real applications of the INSHORE system compared to test performed in controlled environments. Even with the demonstrated difficulty in placing the laser reflection point exactly over the grid marks on the ground, we believe that the obtained results are more significant in the analysis of the overall performance of the INSHORE system in terms of its performance on a sandy shore. This is true for the intrinsic errors of the system and those of the system operation. The background knowledge that we have acquired regarding each of the installed sensors individually supports this statement. The differential GPS positioning of the carrier phase ambiguity fixing technique provides, in the kinematic mode and with restricted to multipath effects, a positioning accuracy bet- 
ter than $2 \mathrm{~cm}$. Additionally, tests performed on the laser sensor in the laboratory reflecting over sandy surfaces showed that its accuracy was below the $1 \mathrm{~cm}$ range. Theoretically, it was expected that the overall system accuracy (not including the system-operation-related errors), in terms of ground point positioning, would be on the range of 2 to $3 \mathrm{~cm}$. This accuracy was confirmed by the validation tests performed. Another point that is worth discussing is the influence of the operator's ability to handle the INSHORE system compared to the intrinsic accuracy of the system. Clearly, the operator must be careful to obtain the highest accuracy. From the analysis provided in "Results", it is evident that the operation-related errors are dominant over the intrinsic errors of the system.

\section{Conclusions}

A new survey system for sandy shore monitoring was proposed, and was named the INSHORE system. The INSHORE system uses a set of GPS receivers and an inertial sensor for additional redundancy and robustness to compute the absolute three-dimensional solution of a point in the system (the phase centre of the outer GPS antenna) and the attitude of the structure holding the sensors. With the antenna structure rigidly attached to a land vehicle and with a range of information on the distance of structure to the ground provided by a laser distance sensor mounted below the outer GPS antenna, the INSHORE system is able to compute the coordinates of the ground points. The positioning data is collected with accuracy of better than $2 \mathrm{~cm}$, independently of the inclination that the structure presents at each instant with respect to a local reference frame, and independently of the survey velocity. Moreover, because the INSHORE system does not have any physical contact point with the ground surface, its survey efficiency is extremely good. Additionally, its operation costs are also low, making the INSHORE system highly appropriate for accurate and low-cost shore monitoring. Four validation tests were performed to assess the accuracy and performance of the INSHORE survey system, confirming its superior accuracy and excellent performance in both static and kinematic modes of operation. The INSHORE survey system is particularly suited for sandy shore monitoring programmes with a particular focus on grid profiles in sub-aerial beach. From these data sources it is possible to generate digital elevation models after interpolation procedures. The sand sample collection in the areas of beach survey can also be performed in an easy way by this land-based mobile platform. Topographic and sedimentary data of surveyed beaches are valuable sources of information that meet the requirements of management interventions and scientific studies.

Acknowledgements This work was developed under the INSHORE project-Integrated System for High Operational Resolution in Shore Monitoring-financed by the Portuguese Science Foundation (FCT-Fundação para a Ciência e a Tecnologia) with funding reference PTDC/ AMB/73169/2006 and co-financed by FEDER funds with funding reference FCOMP-01-0124-FEDER-011869. Paulo Baptista was partially supported by Fundação para a Ciência e a Tecnologia grant SFRH/BPD/63141/2009.

\section{References}

Anders, F. J., \& Byrnes, M. A. (1991). Accuracy of shoreline change rates as determined from maps and aerial photographs. Shore Beach, 59(1), 17-26.

Baptista, P., Bastos, L., Bernardes, C., Cunha, T., \& Dias, J. (2008). Monitoring sandy shore morphologies by DGPS-A practical tool to generate digital elevation models. Journal of Coastal Research, 24(6), 1516-1528.

Baptista, P., Cunha, T., Bernardes, C., Gama, C., Ferreira, Ó., Dias, J. (2010). A precise and efficient methodology to analyse the shoreline displacement rate. Journal of Coastal Research. doi:10.2112/09-1187.1.

Barnard, P., \& Warrick, J. (2010). Dramatic beach and nearshore morphological changes due to extreme flooding at a wave-dominated river mouth. Marine Geology, 271, 131-148.

Dornbusch, U., Moses, C., Robinson, D. A., \& Williams, R. (2008). Soft copy photogrammetry to measure shore platform erosion on decadal timescales. Journal of Coastal Conservation, 11, 193-200.

Emery, K. O. (1961). A simple method of measuring beach profiles. Limnology and Oceanography, 6, 90-93.

Fletcher, C., Rooney, J., Barbee, M., Siang-Chyn, L., \& Richmond, B. (2002). Mapping shoreline change using digital orthophotogrammetry on Maui, Hawaii. Journal of Coastal Research, NS38, 106-124.

Groat, C. G. (2000). U.S. geological survey: Facing the new century. Sea Technology, 41(1), 45-47.

Hansen, J., \& Barnard, P. (2010). Sub-weekly to interannual variability of a high-energy shoreline. Coastal Engineering, 57, 959-972. 
Haxel, J. H., \& Holman, R. A. (2004). The sediment response of a dissipative beach to variations in wave climate. Marine Geology, 206, 73-99.

Hofmann-Wellenhof, B., Lichtenegger, H., \& Collins, J. (2001). GPS: Theory and practice (5th ed.). Wien: Springer.

Holman, R. A., Sallenger, A. H., Lippmann, T. C., \& Haines, J. W. (1993). The application of video image processing to the study of nearshore processes. Oceanography, 6(3), 78-85.

Huising, E. J., \& Gomes Pereira, L. M. (1998). Errors and accuracy estimates of laser data acquired by various laser scanning systems for topographic applications. Journal of Photogrammetry \& Remote Sensing, 53, 245-261.

James, L., \& Farrell, J. L. (1976). Integrated aircraft navigation. New York: Academic Press.

Komar, P. D. (1976). Beach processes and sedimentation. New Jersey: Prentice-Hall.

Lancker, V., Lanckneus, J., Hearn, S., Hoekstra, P., Levoy, F., Miles, J., et al. (2004). Coastal and nearshore morphology, bedforms and sediment transport pathways at Teignmouth (UK). Continental Shelf Research, 24, 1171-1202.

Lippmann, T. C., \& Holman, R. A. (1989). Quantification of sand bar morphology: A video technique based on wave dissipation. Journal of Geophysical Research, 94(C1), 995-1011.

List, J. H., Farris, A. S., \& Sullivan, C. (2006). Reversing storm hotspots on sandy beaches: Spatial and temporal characteristics. Marine Geology, 226, 261279.

Morton, R. A., Leach, M. P., Paine, J. G., \& Cardoza, M. A. (1993). Monitoring beach changes using GPS surveying techniques. Journal of Coastal Research, 9(3), 702720.

Shrestha, R. L., Carter, W. E., Sartori, M., Luzum, B. J., \& Slatton, K. C. (2005). Airborne laser swath mapping: Quantifying changes in sandy beaches over time scales of weeks to years. Journal of Photogrammetry \& Remote Sensing, 59, 222-232.

Wright, L. D., \& Short, A. D. (1984). Morphodynamic variability of surf zones and beaches: A synthesis. Marine Geology, 56, 93-118. 